\title{
Shading-induced failure in thin-film photovoltaic modules: Electrothermal simulation with nonuniformities
}

\author{
M. Nardone ${ }^{\mathrm{a}, *}$, S. Dahal ${ }^{\mathrm{a}}$, J.M. Waddle ${ }^{\mathrm{a}}$ \\ ${ }^{a}$ Department of Physics and Astronomy, Bowling Green State University, Bowling Green, \\ $\mathrm{OH}$ 43403, USA
}

\begin{abstract}
Finite element electrothermal modeling is employed to study shading-induced failure in monolithically integrated thin-film photovoltaic modules. A key element is spatial nonuniformity in current-voltage characteristics, which causes inhomogeneous current flow when part of a module is under reverse bias due to shading. Time-dependent calculations show that spots with lower reverse breakdown voltage experience greater current density and localized thermal runaway that can cause permanent damage, resulting in ohmic shunts. Such failure events lead to performance loss, especially when the module is in normal operating conditions and shunted currents lead to abnormally high module temperatures. Our simulations of temperature distributions, current-voltage characteristics, and electroluminescence are compared to data from the literature for copper indium gallium diselenide devices.
\end{abstract}

Keywords: Numerical simulation, Reverse bias breakdown, Degradation, Monolithic modules, $\mathrm{Cu}(\mathrm{In}, \mathrm{Ga}) \mathrm{Se}$

\section{Introduction}

Thin-film photovoltaic (PV) devices, such as amorphous silicon (a-Si), cadmium telluride (CdTe), and copper indium gallium diselenide (CIGS), have the

\footnotetext{
* Corresponding author

Email address: marcon@bgsu.edu (M. Nardone)
}

Preprint submitted to Solar Energy

October 3, 2016

(C) 2016. This manuscript version is made available under the Elsevier user license http://www.elsevier.com/open-access/userlicense/1.0/ 
distinct cost advantage of large area deposition. By laser scribing, those large areas are divided into individual series-connected cells to form monolithically integrated modules (Hegedus, 2006). A negative side-effect of that process is that nonuniformities are inevitable and can lead to transport and degradation mechanisms that are not as likely in crystalline PV devices (Karpov et al., 2002, 2004a). In addition, the use of bypass diodes to protect against shading-related power loss and degradation is challenging in monolithic modules (Dongaonkar et al., 2013; Lee et al., 2016). When a module is shaded within a series connected system of modules, bypass diodes can prevent the shaded module from experiencing reverse bias. If the bypass diodes open, the shaded module is effectively under short-circuit condition. If the bypass diodes should fail or be otherwise ineffective, the shaded module can experience reverse bias stress, which consumes energy from the other modules and is even more dangerous for the shaded module.

When an illuminated module is partially shaded, the darkened regions can experience reverse bias. It has been observed that CIGS modules subjected to reverse bias stress develop white, worm-like features visible under the glass that were associated with hot-spot ignition (Lee et al., 2016; Westin et al., 2009; Silverman et al., 2015a,b). Random ignition events were identified as correlated voltage and thermography pulses over the course of several minutes under dark, reverse-bias stress (Westin et al., 2009) and by electroluminescence (EL) within seconds during partial shading stress tests (Lee et al., 2016; Silverman et al., 2015a). The effects of such damage were increased shunt conductance and module power loss. The nature of shading-induced degradation in thin-film modules is an open and important problem (Kozinsky et al., 2016).

In this work, we investigate shading-induced failure in thin-film modules using electrothermal numerical simulation. We consider CIGS devices as a case study, but our approach is valid for other types of thin-film modules. Spatial and temporal variations of current and temperature are calculated to better understand the stresses caused by shading and the concomitant reverse bias. The typically observed (Szaniawski et al., 2013; Puttnins et al., 2014) reverse break- 
down characteristic of CIGS devices is included in our model and we argue that the presence of low reverse breakdown voltage points (spatial nonuniformity) is a key factor in shading-induced failure. Our results demonstrate an example of thermal instability as a positive feedback mechanism in a nonuniform thinfilm, the physics of which was described in (Karpov, 2012). In what follows, we distinguish between 'breakdown' and 'failure' by reserving 'breakdown' for the commonly observed increase in current with reverse voltage bias, and 'failure' for the localized, thermal runaway damaging event.

Here we employ the Finite Element Method (FEM) using COMSOL Multiphysics ${ }^{\circledR}$ for quantitative electrothermal analysis of thin-film modules. Cell and module scale electrical simulations based on a network of equivalent circuits are commonly executed using a program such as SPICE (Brecl et al., 2005; Shvydka and Karpov, 2005; Brecl and Topi, 2008; Koishiyev, 2010; Pieters, 2011; Steiner et al., 2011). Electrothermal coupling can also be accomplished using such methods by translating the thermal problem into circuit elements (Maffezzoni and Amore, 2009; Vasko et al., 2014). With FEM, the partial differential equations are discretized by a customizable mesh and recent studies have shown that it can effectively simulate monolithic PV modules (Malm and Edoff, 2008; Lanz et al., 2013; Silverman et al., 2015b). Partial shading of thin-film modules was studied by equivalent circuit simulation for the case of uniform material properties (Dongaonkar et al., 2013). An advantage of the FEM approach is that irregularities in shape, material properties, and external conditions can be easily handled. Moreover, given that nonuniformity is a primary factor in our study, the ability of FEM to easily include localized variations with increased mesh refinement is essential.

\section{Module Simulation Methodology}

\subsection{Electrical Current}

A schematic of one interconnect section of our monolithic module model is shown in Fig. 1 where the lateral current density, $J$, flows through the electrodes 
and is fed by the transverse current densities (lower case $j$ 's). The module model is based on the idea that each cell is comprised of electrical circuit elements connected in parallel to each other via a bottom electrode (back contact, metal) and top electrode [front contact, transparent conductive oxide (TCO)]. In the limit of infinitesimal spacing, current conservation requires that $\nabla \cdot J=Q_{j}$, where $Q_{j}=j / d$ is the current that enters per unit volume of electrode of thickness $d$. From Ohm's Law, $J=-\sigma \nabla \phi$, and the fact that we must consider current flow through both the front and back contacts, we obtain the coupled equations,

$$
\begin{gathered}
\nabla \cdot\left(-\sigma_{f} \nabla \phi_{f}\right)=-j / d_{f}, \\
\nabla \cdot\left(-\sigma_{b} \nabla \phi_{b}\right)=j / d_{b},
\end{gathered}
$$

where $\sigma_{f}$ and $\sigma_{b}$ are the conductivities of the front and back contacts, and $\phi_{f}(x, y)$ and $\phi_{b}(x, y)$ are the electric potential distributions. Conductivity can be obtained from the sheet resistance $\left(R_{\square}\right.$ in units of $\left.\Omega / \square\right)$ by, $\sigma=1 /\left(R_{\square} d\right)$. The coupling is provided by the transverse current, $j$, because any current exiting the back contact must enter the front and vice versa.

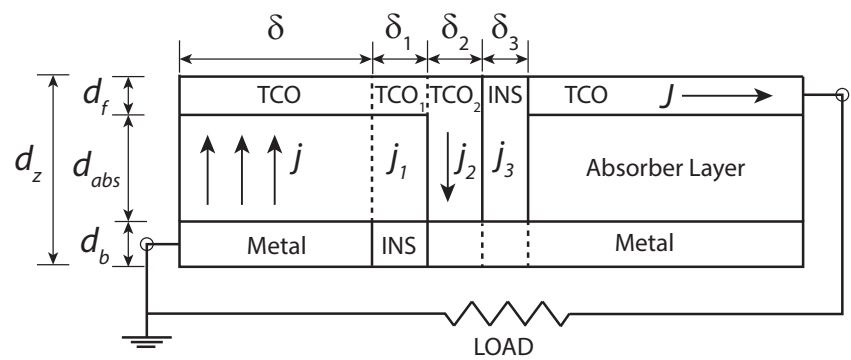

Figure 1: Monolithic module schematic of one interconnect section. Dashed lines indicate current domains, not material breaks. INS is insulating material. Light is incident from the top. This schematic is a simplified representation of a typical substrate configuration device, such as commercial CIGS.

We assume that lateral current through the absorber can be neglected relative to that through the electrodes. This approach allows us to solve two coupled $2 \mathrm{D}$ models rather than one $3 \mathrm{D}$ model with a very large aspect ratio. As shown in 
Fig. 1, one end of the bottom contact is grounded while fixed voltage or current can be applied to the opposite end of the front contact. Given those boundary conditions, one can obtain current-voltage $(I V)$ characteristics by integrating the current density over the boundary area (product of electrode length and $d$ ), which can be done for any contact shape.

Of the four transverse current domains indicated in Fig. 1, $j$ is the diode current, $j_{1}=j_{3}=0$, and $j_{2}=\left(\phi_{f}-\phi_{b}\right) \sigma_{2} / h$ is an ohmic current through the vertical interconnect of conductivity $\sigma_{2}$ (we assume $\sigma_{2}=\sigma_{f}$ ). The diode current is,

$$
j=j_{0} f_{r b}(V)\left[\exp \left(\frac{q V}{A k T}-1\right)\right]-j_{L},
$$

where $q$ is the elementary charge, $V=\phi_{f}-\phi_{b}$ is the local potential difference across device, $A$ is the ideality factor, $k$ is Boltzmann's constant, and $T$ is temperature. We use for the photocurrent $j_{L}=32.5 \mathrm{~mA} / \mathrm{cm}^{2}$ (typical for CIGS at 1-sun light). The function $f_{r b}(V)$ provides the observed reverse breakdown characteristics as described below [cf. Eq. (5)]. We estimate the saturation current as $j_{0}=q G L$ where the $G$ is the generation rate and $L$ is the characteristic collection length of generated carriers. Given that the diffusion length, $L_{d}$, and depletion width, $W_{d}$, are of the same order $\left(L_{d} \approx W_{d} \approx 0.1-1 \mu \mathrm{m}\right)$ for CIGS, we use $L=0.3 \mu \mathrm{m}$. That also implies that most of the generation is within or close to the depletion region, hence $G=n_{i} / \tau$ in the dark, where $n_{i}$ is the intrinsic concentration and $\tau$ is the minority carrier lifetime. With the standard expression for $n_{i}$ the saturation current is given by,

$$
j_{0}=j_{00} \exp \left(-\frac{E_{g}}{2 k T}\right) \quad \text { with } \quad j_{00}=\frac{q L \sqrt{N_{c} N_{v}}}{\tau},
$$

where $E_{g}$ is the band gap, and $N_{c}$ and $N_{v}$ are the effective density of state in the conduction and valence bands. For our purposes, only exponential voltage and temperature dependencies are considered. The physical interpretation of Eq. (4) leads to an ideality factor of $A=2$.

To minimize the number of tunable parameters, the above represents the simplest possible diode model that emphasizes the significant recombination/generation processes that occur in the depletion region of these thin-film heterostructures. 
A more detailed (e.g. two-diode) approach would include generation processes within the quasi-neutral bulk and an ideality factor between 1 and 2, which is typically observed. The presence of a high density of states at the CIGS/CdS interface and accounting for the observed $A>2$ requires consideration of mechanisms that go beyond the two-diode model (Rau, 1999; Nardone et al., 2009). The simple model of Eq. (4) is suitable for the present scope of work but any diode model could be included within our electrothermal module simulation framework.

In this study, all of the parameters in Eq. (4) except for $\tau$ are fixed to reasonable values for CIGS (Gloeckler et al., 2003). The lifetime is varied to account for differences between normal and weak diodes (Karpov et al., 2002), as well as light and dark $J V$ curves under forward bias. For example, Eq. (3) is plotted in Fig. 2 with lifetimes of $\tau=10$ ns and $\tau=5$ ns for a normal diode in the dark and in 1-sun light, respectively, and $\tau=2 \mathrm{~ns}$ for a weak diode in 1-sun light. As lifetime is decreased from $5 \mathrm{~ns}$ to $2 \mathrm{~ns}, V_{o c}$ declines from $0.61 \mathrm{~V}$ to 0.56 $\mathrm{V}$ and efficiency from $14.4 \%$ to $12.8 \%$. That relationship between $\tau$ and $V_{o c}$ is in agreement with experimental findings for CIGS devices (Repins et al., 2010). Overall, the forward bias $J V$ curves are typical for CIGS, including the crossover in light and dark curves at a voltage slightly greater than $V_{o c}$. Results from our detailed semiconductor device simulations using the parameter values from (Gloeckler et al., 2003) are included as points in Fig. 2. It should be noted that a lower lifetime upon exposure to light was assumed to account for the increased non-radiative recombination rate observed in the device simulations. Bias and light dependence of the non-radiative recombination rate and mid-gap center occupation probabilities within the depletion region are included in the device simulation, which accounts for the often observed light/dark cross-over. A lower lifetime in Eq. (4) mimics this effect in the simple diode model employed here.

Also shown in Fig. 2 is the case when Eq. (3) is replaced by $j=V / R_{s h}$, which represents an ohmic shunt of resistance $R_{s h} \approx 10 \mathrm{~m} \Omega \mathrm{cm}^{2}$. The latter value is in agreement with ohmic shunts identified in CIGS modules (Gerber et al., 2015). In our module models, point-like shunts were used with absolute 


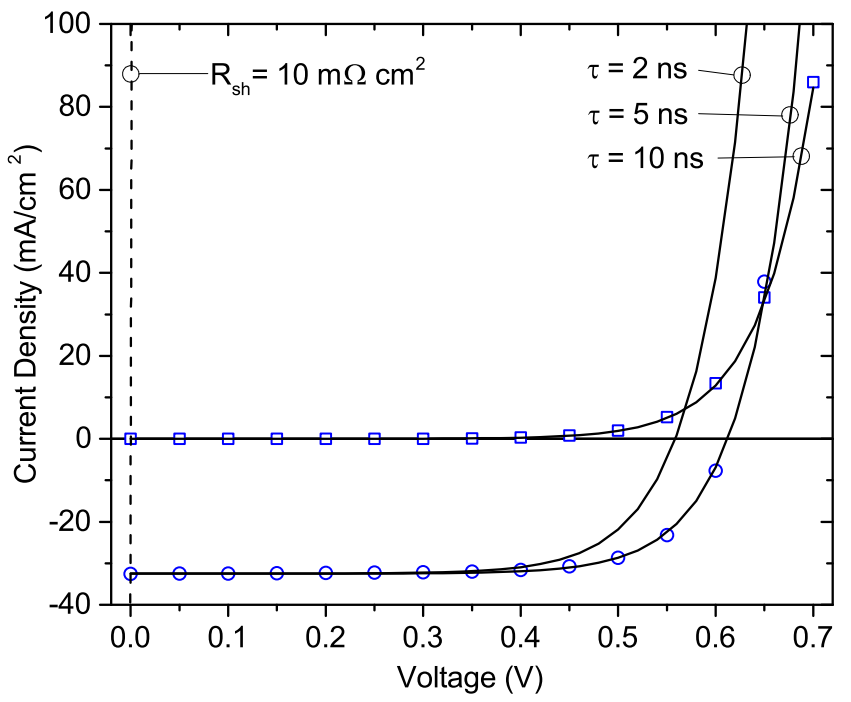

Figure 2: Forward bias $J V$ curves. Solid lines are from Eq. (3) with values from Table 1 and the lifetimes indicated. Open circles (light $J V$ ) and squares (dark $J V$ ) are calculated from semiconductor device modeling. The dashed line is an ohmic shunt with $R_{s h}=10 \mathrm{~m} \Omega \mathrm{cm}^{2}$.

resistances of either $R_{s h}^{0}=50 \Omega$ or $R_{s h}^{0}=10 \Omega$, corresponding to a spatial influence of approximately $100 \mu \mathrm{m}$. Calculations showed the latter value to be a reasonable estimate for shunted modules under forward bias $(V>0 \mathrm{~V})$. However, the lateral decay length of the electric potential in a diode system can range from microns to meters depending on light, voltage, and temperature (Karpov et al., 2004a; Nardone and Karpov, 2008). No series resistance was assumed in the simple diode model or the device simulation. Series resistance was an integral part of the module simulations (detailed below) based on the resistance of the $\mathrm{TCO}$ and metal contacts.

Given our purpose of studying shading effects, it is essential that the diode IV characteristics have the correct features under reverse bias. Experimentally, reverse breakdown voltages of $V_{b r} \approx-4 \mathrm{~V}$ in the dark and $V_{b r} \approx-2 \mathrm{~V}$ in light have been observed in pristine CIGS cells (Szaniawski et al., 2013; Puttnins et al., 2014). That light-enhanced reverse breakdown was found to be most pronounced for blue light rather than red. It was also observed that the reverse 
current strongly increased with increasing temperature for $T>200 \mathrm{~K}$. Because there does not appear to be a satisfactory analytical expression for this phenomenon (further discussion provided in Sec. 4), we used the following function that qualitatively accounts for the data,

$$
f_{r b}= \begin{cases}\exp (-\alpha V), & \text { if } V \leq 0 \\ 1, & \text { otherwise }\end{cases}
$$

Fig. (3) shows the reverse $J V$ curves given by Eqs. (3)-Eq. (5) compared to data points from (Szaniawski et al., 2013). Reasonable, qualitative agreement with both the voltage and temperature dependence of the data (Szaniawski et al., 2013; Puttnins et al., 2014) is obtained for $\alpha=2 \mathrm{~V}^{-1}$ and $\alpha=4 \mathrm{~V}^{-1}$ for the dark and light cases, respectively. The 'defective' case with lower reverse breakdown voltage and $\alpha=6 \mathrm{~V}^{-1}$ was used for local weak spots in our simulations. Such weak spots were identified in local defect regions in CIGS cells (Lee et al., 2016).

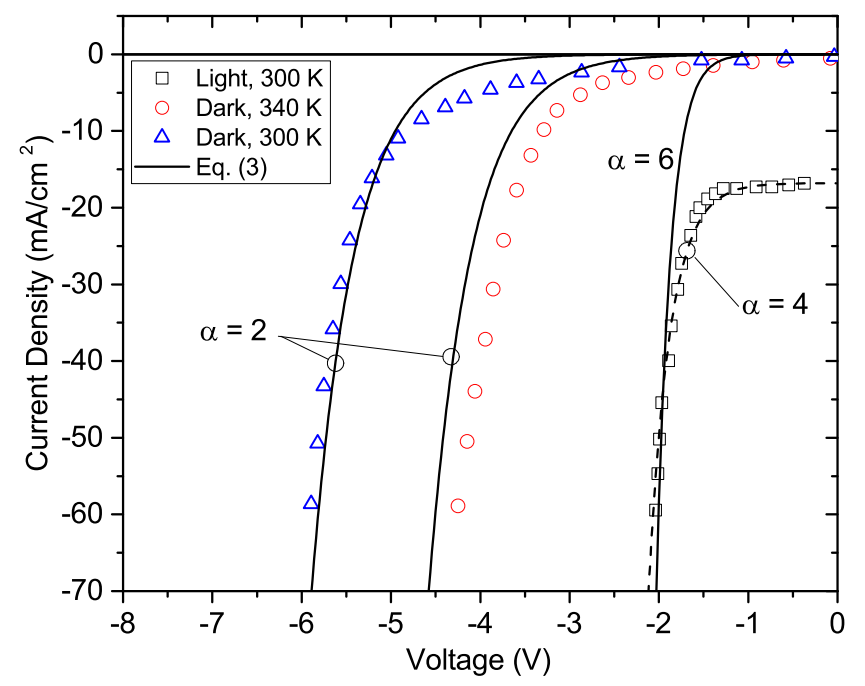

Figure 3: Reverse bias $J V$ curves. Solid lines are from Eqs. (3)-(5) with the values from Table 1 and $\alpha$ as indicated. Points are data extracted from (Szaniawski et al., 2013). The light $J V$ curve (dashed) uses $j_{L}=-16.8 \mathrm{~mA} / \mathrm{cm}^{2}$ to match experimental conditions. 


\subsection{Thermal Physics}

The temperature distribution is governed by,

$$
\rho C \frac{\partial T}{\partial t}+\nabla \cdot(-\kappa \nabla T)=Q_{h} / d_{z}
$$

where $\kappa$ is the thermal conductivity, $\rho$ is the density, and $C$ is the specific

heat capacity of the various materials. By neglecting temperature differences in the transverse direction over the several micron thick device layer $\left(d_{z}=4 \mu \mathrm{m}\right.$ total thickness), we consider the module to be a $2 \mathrm{D}$ domain and solve for the temperature distribution, $T(x, y)$. A 3D model including packaging material can also be used (Silverman et al., 2015b). The source term, $Q_{h}$, is given by,

$$
Q_{h}=Q_{L}+j V+d(\mathbf{J} \cdot \mathbf{E})-h\left(T-T_{\infty}\right)-\varepsilon \sigma\left(T^{4}-T_{\infty}^{4}\right) .
$$

Power deposited by the light source is $Q_{L}\left(1000 \mathrm{~W} / \mathrm{m}^{2}\right.$ at $\left.1 \mathrm{sun}\right), j V$ is due to the diodes or shunts (negative when power is dissipated in an external load), the third term is Joule heating in the front and back contacts of thickness $d=d_{f}$ or $d=d_{b}, h$ is the convective heat transfer coefficient, $T_{\infty}$ is the ambient temperature, $\varepsilon$ is the emissivity, and $\sigma$ is the Stefan-Boltzmann constant. We use $\varepsilon=0$ for the back contact metal and $\varepsilon=1$ otherwise. In our models, radiative and convective cooling are greater for unencapsulated modules than for encapsulated ones, as discussed in Sec. 3. Boundary conditions along the outer edges of the domain were set to convective and radiative heat flux, similar to the last two terms in Eq. (7).

\subsection{Numerical Methods and Parameter Values}

Eqs. (1)-(7) were solved as a coupled system for $\phi_{f}(x, y), \phi_{b}(x, y)$, and $T(x, y)$. All calculations were conducted by FEM using COMSOL Multiphysics ${ }^{\circledR}$. We used as our test case a 10x10 $\mathrm{cm}^{2}$ monolithic CIGS mini-module with 20, 5 -mm wide cells connected in series. The $2 \mathrm{D}$ domains were discretized by triangular mesh elements in the cell areas and quadrilateral elements in the scribe areas to better handle the large differences in widths. Overall, approximately 10000-20000 mesh elements were used to discretize the mini-module and the 
mesh was highly refined around shunt or weak diode points, as shown in Fig. 4. The Newton iteration method was employed to solve the system of equations, along with backward differentiation formulas for time-dependent studies. Parameter values are provided in Table 1. The insulation material in Table 1 corresponds to the scribe insulation between cells, not any encapsulant materials. Simple models of radiative cooling and convection embody the thermal effects [see Eq. (7)] of the encapsulant materials, which allow one to use a 2D rather than 3D model.

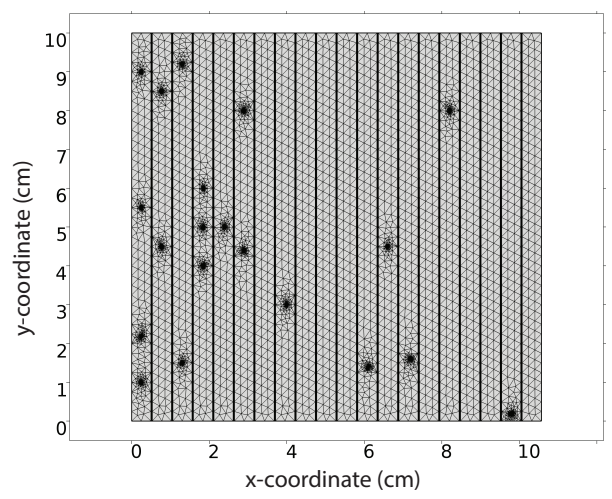

Figure 4: Meshed domain of a mini-module with 20 randomly-placed points of nonuniformity.

EL intensity, $\phi_{E L}$, was simulated by using the relation $\phi_{E L} \propto \exp (q V / k T)$, where $V$ is the voltage across the junction (Rau, 2007). A more complete method combining EL, lock-in thermography, and simulation enables quantitative analysis of module parameters, such as spatial voltage distributions, and series and shunt resistances (Gerber et al., 2015).

\section{Results}

We consider first the case of a uniform module. The calculated forwardbias, 1-sun light $I V$ curve of the CIGS mini-module at a fixed temperature of $T=293 \mathrm{~K}$ is shown in Fig. 5(a) [metrics: $\eta$ (efficiency) $=13.5 \%, V_{o c}=$ $\left.12.2 \mathrm{~V}, I_{s c}=0.162 \mathrm{~A}, F F=68.4 \%\right]$. Fully shading 4 of the 20 cells $(20 \%$ shaded module) results in the electric potential distribution $\left(\phi_{f}-\phi_{b}\right)$ shown in 


\begin{tabular}{cccc}
\hline \hline Parameter & Unit & Values & Ref. \\
\hline$R_{\square f}, R_{\square b}$ & $\Omega / \square$ & 10,1 & (Silverman et al., 2015b) \\
$\sigma_{I N S}$ & $\Omega^{-1} m^{-1}$ & $10^{-10}$ & (Silverman et al., 2015b) \\
$d_{f}, d_{b}$ & $\mu \mathrm{m}$ & 1,1 & NA \\
$E_{g}$ & $\mathrm{eV}$ & 1.2 & (Gloeckler et al., 2003) \\
$N_{c}$ & $\mathrm{~cm}^{-3}$ & $2.2 \times 10^{18}$ & (Gloeckler et al., 2003) \\
$N_{v}$ & $\mathrm{~cm}^{-3}$ & $1.8 \times 10^{19}$ & (Gloeckler et al., 2003) \\
$\kappa_{f}, \kappa_{b}, \kappa_{I N S}$ & $\mathrm{~W} /(\mathrm{m} \mathrm{K})$ & $100,100,1$ & (Haynes, 2014) \\
$\rho_{f}, \rho_{b}, \rho_{I N S}$ & $\mathrm{~kg} / \mathrm{m}^{3}$ & $10^{4}, 10^{4}, 10^{4}$ & (Haynes, 2014) \\
$C_{f}, C_{b}, C_{I N S}$ & $\mathrm{~J} /(\mathrm{kg} \mathrm{K})$ & $250,250,250$ & (Haynes, 2014) \\
$h$ & $\mathrm{~W} /\left(\mathrm{m}^{2} \mathrm{~K}\right)$ & 20 & (Silverman et al., 2015b) \\
$d_{z}$ & $\mu \mathrm{m}$ & 4 & NA \\
cell pitch & $\mathrm{mm}$ & 5 & (Silverman et al., 2015b) \\
\hline
\end{tabular}

Table 1: Input parameter values. Subscripts $f, b$, and $I N S$ refer to front, back, and insulation, respectively. $R_{\square}$ - sheet resistance, $\sigma_{I N S}$ - electrical conductivity of insulation, $d$ - thickness of contact, $E_{g}$ - bandgap, $N_{c}$ and $N_{v}$ - effective density of states in conduction and valence bands, $\kappa$ - thermal conductivity, $\rho$ - density, $C$ - specific heat capacity, $d_{z}$ - device thickness, $h$ - convective heat transfer coefficient. Order of magnitude values were estimated from (Haynes, 2014). Values for $\tau$ and $\alpha$ are provided in Figs. 2 and 3, respectively.

Fig. 5(b) where the light intensity for the shaded portion is $0 \mathrm{~W} / \mathrm{m}^{2}$ and 1000 $\mathrm{W} / \mathrm{m}^{2}$ otherwise. Illuminated cells have a junction potential of $0.6 \mathrm{~V}$ while the shaded cells have $-2.4 \mathrm{~V}$, resulting in an overall module voltage of $V=0 \mathrm{~V}$. Such large negative voltages across the shaded cells can lead to junction electric fields on the order of $10^{5} \mathrm{~V} / \mathrm{cm}$ and related breakdown effects (Karpov et al., 2004b). The $I V$ curve for this scenario is shown in Fig. 5(a) along with that of the fully illuminated module. The partially shaded module shows a very low short-circuit current of $I_{s c}=-0.16 \mathrm{~mA}$ due to the blocking but slightly leaky nature of the darkened cells.

Next we consider the same shading scenario in the presence of 20 randomly placed weak spots ( $\alpha=6$ in Fig. 3) at the locations indicated in Fig. 4, with 

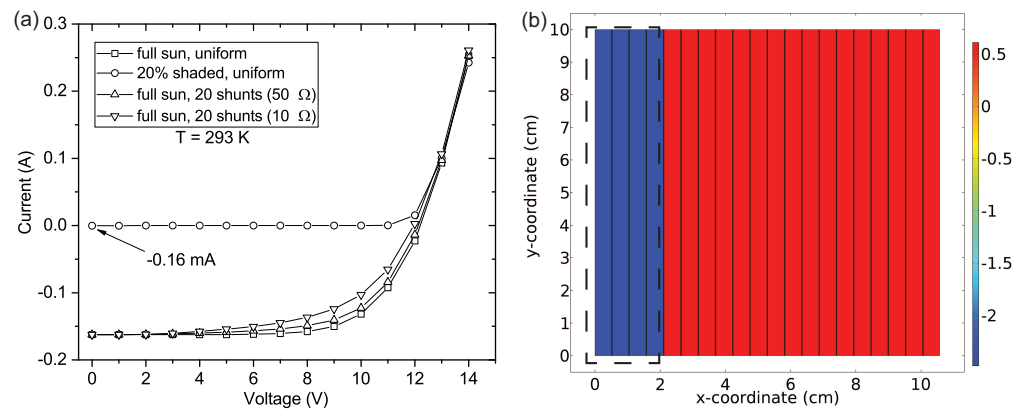

Figure 5: (a) Module $I V$ calculated at $T=293 \mathrm{~K}$ with the conditions indicated in the legend. (b) Local junction electric potential (V) for a $20 \%$ shaded CIGS mini-module. Dashed box indicates fully shaded area and the rest is under $1000 \mathrm{~W} / \mathrm{m}^{2}$ light intensity.

and without electrothermal coupling. The presence of such a low density of the weak diodes has a negligible effect on the $I V$ curve when measured at standard conditions (not shown). However, when the module is partially shaded and temperature dependence is included, the weak spots that experience negative bias pass a larger current due to their lower reverse breakdown voltage, resulting in substantial heating.

We consider heat transfer for two cases: 1) an unecapsulated module with radiative cooling and strong convection $\left[h=20 \mathrm{~W} /\left(\mathrm{m}^{2} \mathrm{~K}\right)\right]$; and 2$)$ an encapsulated module with radiative cooling omitted and weak convection $[h=2$ $\left.\mathrm{W} /\left(\mathrm{m}^{2} \mathrm{~K}\right)\right]$. The thermal model for the unencapsulated cell does not include a glass substrate, which is always present unless a flexible, thermally conductive substrate is used. Presence of the glass substrate would reduce the radiative and convective cooling and could result in a vertical temperature gradient along the cell edges. Fig. 6 shows our simulated temperature distributions for the $20 \%$ shaded, unencapsulated module at $100 \mathrm{~s}$ and $600 \mathrm{~s}$ after switching on the 1-sun light source with ambient $T_{\infty}=293 \mathrm{~K}$ and maintaining a constant $V=0$ $\mathrm{V}$ (short-circuit condition). Heating of the unshaded area to a normal operating temperature of $T \approx 312 \mathrm{~K}$ requires about $100 \mathrm{~s}$. After that, the steady-state condition is attained near $600 \mathrm{~s}$ with localized temperatures reaching $T \approx 700 \mathrm{~K}$. Note that temperatures in the shaded part of the module can increase to values 
greater than in the illuminated part due to the Joule heating effect of the weak spots. Also, clustering of high temperature spots is evident in Fig. 6 at 600 $\mathrm{s}$, which is likely due to the close proximity and interaction of the three spots in the top left corner. The short-circuit current at $100 \mathrm{~s}, I_{s c}=-0.36 \mathrm{~mA}$, is similar to the uniform case [see Fig. 5(a)], but it increases to $I_{s c}=-0.138 \mathrm{~A}$ by $600 \mathrm{~s}$ indicating that most of the current in the shaded cells is flowing through the weak spots. Very similar thermal and $I V$ measurements were reported in (Lee et al., 2016) for $20 \%$ shaded CIGS modules. In that work it was determined that the bypass diode failed to alleviate current flow through the module when most of it flowed through the weak spots identified by thermal imaging.
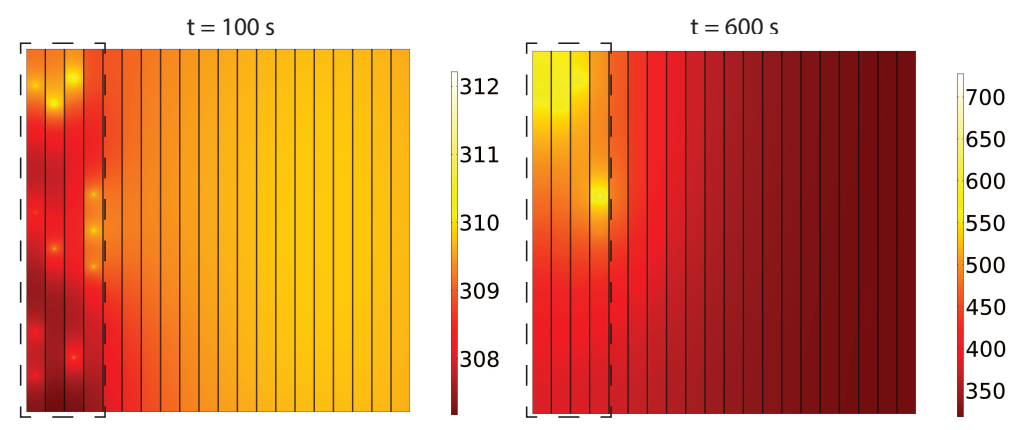

Figure 6: Temperature distributions (in K) in a $20 \%$ shaded, unencapsulated mini-module with 20 non-ohmic shunts ( $\alpha=6$ in Fig. 3) at $100 \mathrm{~s}$ and $600 \mathrm{~s}$ after turning on the light source of $1000 \mathrm{~W} / \mathrm{m}^{2}$ intensity. Dashed box delineates shaded region. Note the difference in scales.

It is interesting to study the evolution of the temperature. Fig. 7 plots the maximum temperature of the module as a function of time. For the unencapsulated case, rapid thermal runaway is visible at $t=490 \mathrm{~s}$ and at $t=100 \mathrm{~s}$ for the encapsulated case. In both cases, the temperature at the runaway point is $T \approx 350 \mathrm{~K}$. Quantitative comparison of the heating terms in Eq. 7 indicated that the largest contribution at the runaway point was due to joule heating, $J^{2} / \sigma_{f}$, in the TCO layer with current density on the order of $\sim 10^{8} \mathrm{~A} / \mathrm{m}^{2}$ in the $1 \mu \mathrm{m}$-thick electrode (see inset of Fig. 7). The post-runaway cooling is due to the large thermal gradient. Based on the numerical analysis, the temperature 
decays over a radius of order $r \sim 1 \mathrm{~cm}$ at the runaway event. As conduction then dominates in Eq. (6), the characteristic decay time of the temperature is given by $\tau=r^{2} / D$, where the thermal diffusivity is $D=\kappa /(C \rho)$. Using the thermal properties of TCO given in Table I results in $\tau$ on the order of 1-10 s. This is only a rough estimate, however, because there is a continuing thermal source at the weak spot and competing cooling mechanisms. When the heating and cooling terms balance, $\partial T / \partial t \sim 0$ and saturation temperature is achieved.

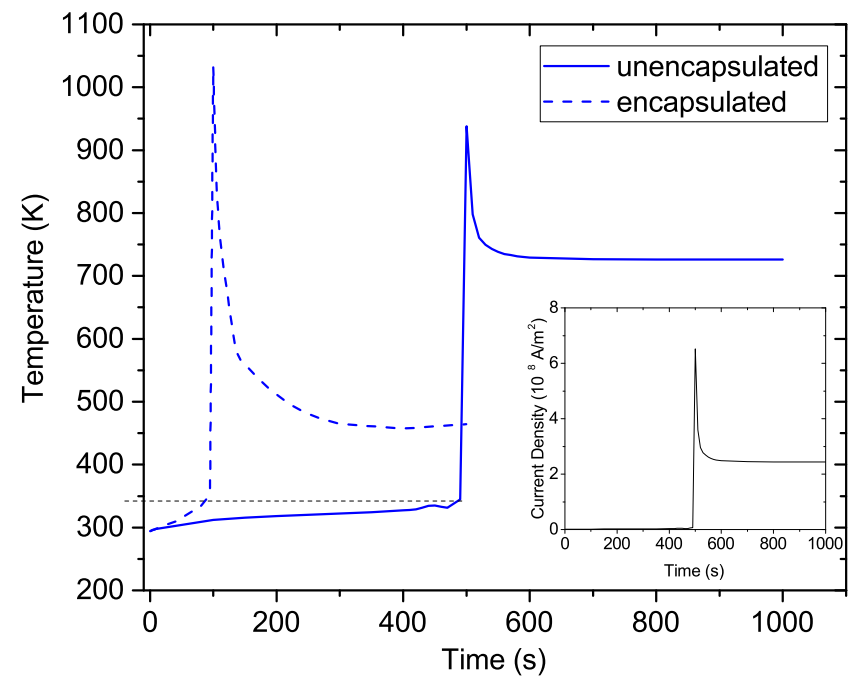

Figure 7: Maximum module temperature as a function of time. Horizontal dashed line indicates temperature at the runaway event. Inset: maximum current density in the TCO layer as a function of time.

For the purposes of the calculations shown in Fig. 7, it was assumed that the material and electronic properties of the device were not changed by the thermal runaway event. It is likely though that such an event would cause irreversible damage and possibly create ohmic shunts. In our subsequent calculations, we assume that those hot spots were converted to ohmic shunts after thermal breakdown, as described below. The actual transition from non-ohmic to ohmic shunts is beyond the present scope.

The explosive, localized heating could lead to the observed (Lee et al., 2016; Westin et al., 2009; Silverman et al., 2015b,a) material damage and ohmic shunt 
formation. Ohmic shunts will certainly have a detrimental impact on performance. We calculated the effects of 20 ohmic shunts with $R_{s h}^{0}=10 \Omega$ and 50 $\Omega$ at the same locations as the weak spots considered above. Omitting thermal aspects and assuming a fixed temperature of $T=293 \mathrm{~K}$ leads to the $I V$ curves shown in Fig. 5. The efficiency decreased from $13.5 \%$ for the uniform module to $12.6 \%$ and $11.1 \%$, respectively for the $50 \Omega$ and $10 \Omega$ cases. Most of the loss was through fill-factor, followed by $V_{o c}$. Module shunt resistance [measured as $(d I / d V)^{-1}$ at $\left.V=0\right]$ decreased significantly from $3 \times 10^{5} \Omega$ in the uniform case to $1 \times 10^{5} \Omega$ and $8 \times 10^{3} \Omega$ for the $50 \Omega$ and $10 \Omega$ cases, respectively. These results are consistent with measurements (Lee et al., 2016; Silverman et al., 2015a).

It is misleading, however, to consider the effects of shunts at a constant temperature only. Simulating field conditions by allowing the temperature to evolve to steady-state in an ambient $T_{\infty}=293 \mathrm{~K}$ and $1000 \mathrm{~W} / \mathrm{m}^{2}$ light intensity (normal operating conditions, NOC) with ohmic shunts present, results in a significant power loss and localized heating in the unencapsulated module, as shown in Figs. 8 and 9(b), respectively. For a uniform module, the $I V$ curves in Fig. 8 indicate that $V_{o c}$ decreased from $12.2 \mathrm{~V}$ at $T=293 \mathrm{~K}$ to $11.4 \mathrm{~V}$ at NOC; temperature near the maximum power point (MPP) was a nearly uniform 312 K. With the 20, $10 \Omega$ shunts, $V_{o c}$ decreased to $7.8 \mathrm{~V}$ and localized temperatures increased to $T>450 \mathrm{~K}$ near MPP of the uniform module, as shown by the right axis in Fig. 8.

EL simulation for the case of 20 ohmic shunts with $R_{s h}^{0}=10 \Omega$, constant module temperature $T=293 \mathrm{~K}$, and forward bias of $12 \mathrm{~V}$ in the dark is shown in Fig. 9(a). Steady-state temperature for the same shunting conditions at 8 V forward bias and 1-sun light intensity is shown in Fig. 9(b). Similar EL and thermography features have been observed after periods of shading stress on the order of seconds (Lee et al., 2016; Silverman et al., 2015a). Our prediction of $t=100-500 \mathrm{~s}$ for the thermal runaway event may be larger than the observed values because our simulation was initiated in the dark condition while in the experiments shade was cast after the module warmed up to a stable temperature in the light. 


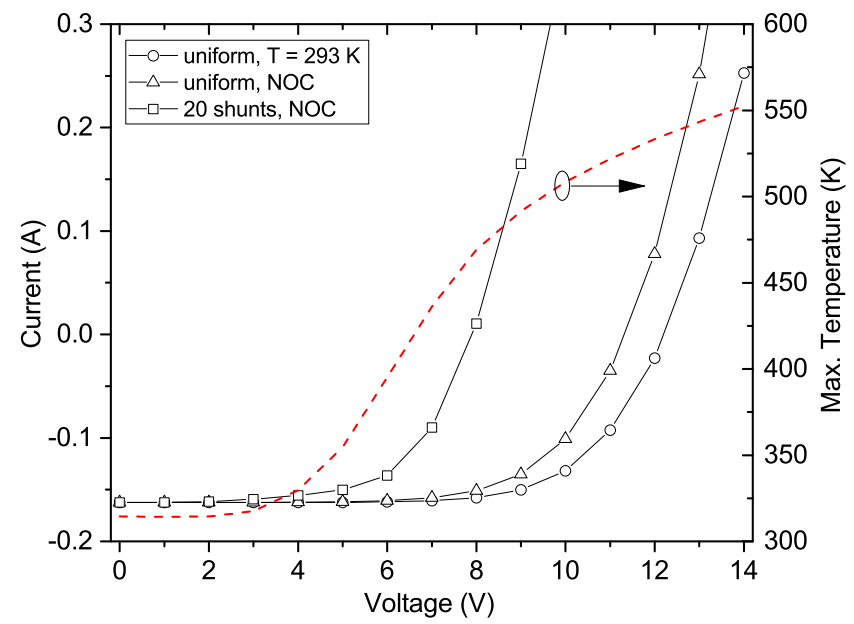

Figure 8: Simulated IV curves for a uniform unencapsulated module at constant $T=293 \mathrm{~K}$, at normal operating conditions (NOC) (ambient $T_{\infty}=293 \mathrm{~K}, 1$-sun light), and a module with $20,10 \Omega$ shunts at NOC. Right axis is the steady-state maximum temperature in the shunted module.

\section{Discussion}

We elected to use Eq. (5) for the reverse IV characteristics of a CIGS device because it provides the voltage, temperature, and light dependencies that are in qualitative agreement with the data (Szaniawski et al., 2013; Puttnins et al., 2014). That was essential for the proper simulation of electrothermal effects with nonuniformities and shading. Determination of the most appropriate physical model requires further investigation because there are a few possible candidates. At the temperatures of interest here, avalanche multiplication would occur at breakdown voltages greater than those observed for CIGS and the significant scattering processes in this polycrystalline material make it quite unlikely (Sze and Ng, 2006). Direct, band-to-band tunneling is often considered a possibility when $V_{b r} \lesssim 4 E_{g} / q$ and the temperature coefficient of $V_{b r}$ is negative, which is the case here. However, it is unlikely that high enough doping concentrations are achieved in CIGS to create a sufficiently large field and thin energy barrier. It was shown that a Poole-Frenkel mechanism could reproduce the observed 


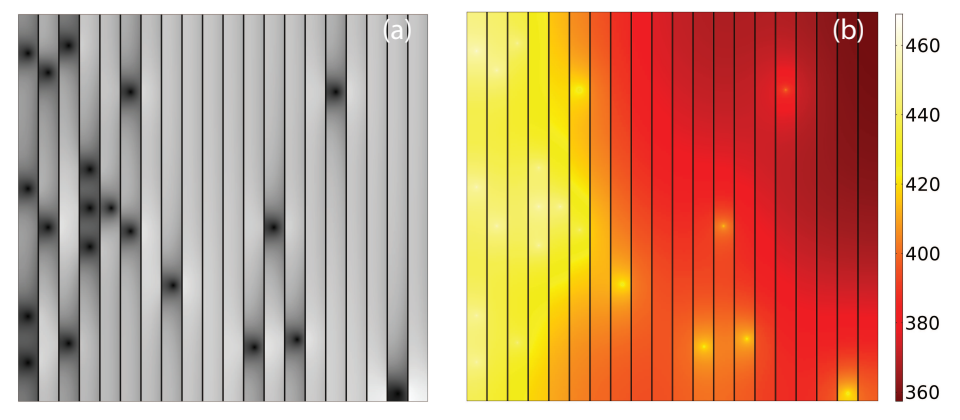

Figure 9: Simulations of (a) EL at $12 \mathrm{~V}$ forward bias in the dark and (b) temperature distribution at $8 \mathrm{~V}$ forward bias fully illuminated at 1-sun light (b). 20 ohmic shunts with $R_{\text {sh }}^{0}=10$ $\Omega$ are active in an unencapsulated module.

temperature and light dependence of the reverse JV characteristics of CIGS cells (Silverman et al., 2015b; Sun et al., 2015). Trap-assisted tunneling (Hurkx et al., 1992) and hopping conduction (Nardone et al., 2009) are also candidate mechanisms that require further quantitative analysis. Another possibility is the presence of semi-shunts, which are conductive filaments that extend part-way through the absorber material (Karpov and Shvydka, 2014). Field enhancement and tunneling at the tip of the semi-shunts can lead to reverse breakdown current that is exponential in voltage.

Our model only considered two possible reverse breakdown characteristics ( $\alpha=2$ and $\alpha=6$ ) to demonstrate the effects of nonuniformity in a simple way. In reality, the inhomogeneities would be better represented by a range of $\alpha$ values that would lead to a broad distribution of currents due to the exponential form of the $J(V)$ function. However, the exponential nature of the diode characteristic causes the system behavior to be driven by the unlikely yet very influential weakest spots (Shvydka and Karpov, 2005). An important question pertains to whether the weak spots were present prior to shading or if they were created by the electric field under reverse bias. Moreover, recent work (Vasko et al., 2014) showed that runaway hot spots can arise spontaneously, even without material nonuniformity, due to thermal inhomogeneities with higher temperatures often close to busbars. 
Recent theoretical work (Karpov, 2012) on thermal instabilities in thin-film semiconductors provided an overheat criteria of $\Theta=\delta T \bar{E} /\left(k T_{0}^{2}\right) \approx 4-5$, above which thermal breakdown is possible. Using values of diode activation energy, $\bar{E}=1 \mathrm{eV}$, and average temperature, $T_{0}=300 \mathrm{~K}$, yields a critical temperature fluctuation of $\delta T \approx 30-50 \mathrm{~K}$, in good agreement with our result shown in Fig. 7. That criterion is based on the concept that the instability occurs when the rate of heat input surpasses the rate of cooling by a sufficient degree in a system where the electrical conductivity increases exponentially with temperature. Our quantitative comparison of the terms in Eq. (7) confirmed that the runaway event occurred when the convective cooling was overtaken by the joule heating terms. More exact comparison to theory is difficult because in our case constant input power is not maintained; the module is held at the short-circuit condition prior to the runaway event, but local voltages and currents are allowed to vary and balance the system voltage, We used short-circuit conditions to mimic experimental conditions in (Lee et al., 2016).

The model provides a quantitative analysis of the time dependence that may be useful for developing laboratory stress tests for shading-induced failure. Also, mitigation measures could be simulated, such as improved bypass diode concepts and the effects of reducing nonuniformity, possibly through the use of interfacial layer treatments (Roussillon et al., 2004).

\section{Conclusions}

Thin-film photovoltaic modules that are subjected to partial shading can exhibit rapid degradation. Electrothermal analysis indicates that the kinetic mechanism is based on weak spots with low reverse breakdown voltage that can experience explosive thermal runaway. Permanent damage could lead to ohmic shunting and excessive long-term heating with concomitant performance loss under normal operating conditions. 


\section{Acknowledgments}

This paper was developed based upon funding from the Alliance for Sustainable Energy, LLC, Managing and Operating Contractor for the National Renewable Energy Laboratory for the U.S. Department of Energy.

\section{References}

Brecl, K., Topi, M., 2008. Simulation of losses in thin-film silicon modules for different configurations and front contacts. Progress in Photovoltaics: Research and Applications 16 (6), 479-488.

URL http://onlinelibrary.wiley.com/doi/10.1002/pip.831/full

Brecl, K., Topi, M., Smole, F., 2005. A detailed study of monolithic contacts and electrical losses in a large-area thin-film module. Progress in Photovoltaics: Research and Applications 13 (4), 297-310.

URL http://onlinelibrary.wiley.com/doi/10.1002/pip.589/full

Dongaonkar, S., Deline, C., Alam, M. A., 2013. Performance and reliability implications of two-dimensional shading in monolithic thin-film photovoltaic modules. IEEE Journal of Photovoltaics 3 (4), 1367-1375.

URL http://ieeexplore ieee . org / xpls / abs_all . jsp? arnumber $=$ 6558752

Gerber, A., Huhn, V., Tran, T. M. H., Siegloch, M., Augarten, Y., Pieters, B. E., Rau, U., Apr. 2015. Advanced large area characterization of thin-film solar modules by electroluminescence and thermography imaging techniques. Solar Energy Materials and Solar Cells 135, 35-42.

URL http : / / www . sciencedirect . com / science / article / pii / S0927024814005029

Gloeckler, M., Fahrenbruch, A. L., Sites, J. R., 2003. Numerical modeling of CIGS and CdTe solar cells: setting the baseline. In: Photovoltaic Energy Conversion, 2003. Proceedings of 3rd World Conference on. Vol. 1. IEEE, pp. 
491-494.

URL http : / / ieeexplore ieee .org / xpls / abs_all . jsp? arnumber $=$ 1305328

Haynes, W. M., 2014. CRC handbook of chemistry and physics. CRC press.

URL https : / / books . google . com / books ? hl = en \& lr = \&id = bNDMBQAAQBAJ \&o $i=$ fnd\&pg $=P P 1 \& d q=C R C+$ handbook\&ots $=H 7$ cBsqwEWL $\&$ sig $=$ efUwneVM7vj5ny-PS3qx0_q5bxc

Hegedus, S., 2006. Thin film solar modules: the low cost, high throughput and versatile alternative to Si wafers. Progress in Photovoltaics: Research and Applications 14 (5), 393-411.

URL http://onlinelibrary.wiley.com/doi/10.1002/pip.704/full

Hurkx, G. A. M., Klaassen, D. B. M., Knuvers, M. P. G., 1992. A new recombination model for device simulation including tunneling. Electron Devices, IEEE Transactions on 39 (2), 331-338.

URL http://ieeexplore.ieee.org/xpls/abs_all.jsp?arnumber $=121690$

Karpov, V. G., 2012. Coupled electronheat transport in nonuniform thin film semiconductor structures. Physical Review B 86 (16), 165317.

URL http://journals . aps . org/prb/abstract/10.1103/PhysRevB. 86. 165317

Karpov, V. G., Compaan, A. D., Shvydka, D., 2002. Effects of nonuniformity in thin-film photovoltaics. Applied Physics Letters 80 (22), 4256-4258.

URL http://scitation.aip.org/content/aip/journal/apl/80/22/10. 1063/1.1483118

Karpov, V. G., Compaan, A. D., Shvydka, D., 2004a. Random diode arrays and mesoscale physics of large-area semiconductor devices. Physical Review B 69 (4), 045325.

URL http://journals . aps .org/prb/abstract/10.1103/PhysRevB. 69 . 045325 
Karpov, V. G., Shvydka, D., Aug. 2014. Semi-shunt field emission in electronic devices. Applied Physics Letters 105 (5), 053904.

URL http://scitation.aip.org/content/aip/journal/apl/105/5/10. $1063 / 1.4892547$

Karpov, V. G., Shvydka, D., Roussillon, Y., Oct. 2004b. $\$\{E\}^{\wedge}\{2\} \$$ phase transition: Thin-film breakdown and Schottky-barrier suppression. Physical Review B 70 (15), 155332.

URL http://link.aps.org/doi/10.1103/PhysRevB.70.155332

Koishiyev, G. T., 2010. Analysis of impact of non-uniformities on thin-film solar cells and modules with 2-D simulations. Ph.D. thesis, Colorado State University.

URL http:// physics . colostate . edu / groups / photovoltaic/PDFs / Galym\%20Thesis.pdf

Kozinsky, I., Bob, B., Jones-Albertus, R., Jun. 2016. Challenges and Opportunities for Improving Thin-Film Photovoltaics. MRS Advances FirstView, 1-6. URL http://journals.cambridge.org/article_S2059852116004369

Lanz, T., Bonmarin, M., Stuckelberger, M., Schlumpf, C., Ballif, C., Ruhstaller, B., 2013. Electrothermal finite-element modeling for defect characterization in thin-film silicon solar modules. Selected Topics in Quantum Electronics, IEEE Journal of 19 (5), 1-8.

URL http://ieeexplore ieee . org / xpls / abs_all . jsp? arnumber = 6472010

Lee, J. E., Bae, S., Oh, W., Park, H., Kim, S. M., Lee, D., Nam, J., Mo, C. B., Kim, D., Yang, J., Kang, Y., Lee, H.-s., Kim, D., Jan. 2016. Investigation of damage caused by partial shading of CuInxGa(1-x)Se2 photovoltaic modules with bypass diodes. Progress in Photovoltaics: Research and Applications, $\mathrm{n} / \mathrm{a}-\mathrm{n} / \mathrm{a}$.

URL http : / / onlinelibrary · wiley . com / doi / 10 . 1002 / pip . 2738 / abstract 
Maffezzoni, P., Amore, D. D., 2009. Compact electrothermal macromodeling of photovoltaic modules. Circuits and Systems II: Express Briefs, IEEE Transactions on 56 (2), 162-166.

URL http : / / ieeexplore ieee .org / xpls / abs_all . jsp? arnumber $=$ 4785142

Malm, U., Edoff, M., 2008. Influence from front contact sheet resistance on extracted diode parameters in CIGS solar cells. Progress in Photovoltaics: Research and Applications 16 (2), 113-121.

URL http://onlinelibrary.wiley.com/doi/10.1002/pip.784/full

Nardone, M., Karpov, V. G., 2008. Admittance characterization of semiconductor junctions. Journal of Applied Physics 103 (8), 084508.

URL http://scitation. aip.org/content/aip/journal/jap/103/8/10. $1063 / 1.2903142$

Nardone, M., Karpov, V. G., Shvydka, D., Attygalle, M. L. C., 2009. Theory of electronic transport in noncrystalline junctions. Journal of Applied Physics 106 (7), 074503.

URL http://scitation.aip.org/content/aip/journal/jap/106/7/10. $1063 / 1.3213336$

Pieters, B. E., Jul. 2011. Spatial modeling of thin-film solar modules using the network simulation method and SPICE. IEEE Journal of Photovoltaics 1 (1), 93-98.

Puttnins, S., Jander, S., Wehrmann, A., Benndorf, G., Stlzel, M., Mller, A., von Wenckstern, H., Daume, F., Rahm, A., Grundmann, M., 2014. Breakdown characteristics of flexible $\mathrm{Cu}(\mathrm{In}, \mathrm{Ga}) \mathrm{Se} 2$ solar cells. Solar Energy Materials and Solar Cells 120, 506-511.

URL http : / / ww . sciencedirect . com / science / article / pii / S0927024813005096

Rau, U., Jan. 1999. Tunneling-enhanced recombination in $\mathrm{Cu}(\mathrm{In}, \mathrm{Ga}) \mathrm{Se}[\mathrm{sub} 2]$ heterojunction solar cells. Applied Physics Letters 74 (1), 111. 
URL http : / / ezproxy . bgsu . edu : 8080/ login? url =http : / / search . ebscohost $\cdot$ com $/$ login $\cdot$ asp $x$ direct $=$ true $\& d b=i i h \& A N=4290666 \&$ site $=$ ehost-live\&scope=site

Rau, U., Aug. 2007. Reciprocity relation between photovoltaic quantum efficiency and electroluminescent emission of solar cells. Physical Review B $76(8), 085303$.

URL http://link.aps.org/doi/10.1103/PhysRevB.76.085303

Repins, I. L., Metzger, W. K., Perkins, C. L., Li, J. V., Contreras, M. A., Nov. 2010. Correlation Between Measured Minority-Carrier Lifetime and Device Performance. IEEE Transactions on Electron Devices 57 (11), 2957-2963.

Roussillon, Y., Giolando, D. M., Shvydka, D., Compaan, A. D., Karpov, V. G., 2004. Blocking thin-film nonuniformities: Photovoltaic self-healing. Applied Physics Letters 84 (4), 616-618.

URL http://scitation.aip.org/content/aip/journal/apl/84/4/10. 1063/1.1644049

Shvydka, D., Karpov, V. G., 2005. Power generation in random diode arrays. Physical Review B 71 (11), 115314.

URL http://journals . aps . org/prb/abstract/10.1103/PhysRevB. 71 . 115314

Silverman, T. J., Deceglie, M. G., Deline, C., Kurtz, S., 2015a. Partial shade stress test for thin-film photovoltaic modules. In: SPIE Optics+ Photonics for Sustainable Energy. International Society for Optics and Photonics, pp. 95630F-95630F.

URL http://proceedings . spiedigitallibrary . org/proceeding . aspx? articleid $=2446460$

Silverman, T. J., Deceglie, M. G., Sun, X., Garris, R. L., Alam, M. A., Deline, C., Kurtz, S., 2015b. Thermal and electrical effects of partial shade in monolithic thin-film photovoltaic modules. Photovoltaics, IEEE Journal of 5 (6), 
$1742-1747$.

URL http : / / ieeexplore ieee .org / xpls / abs_all . jsp? arnumber $=$ 7286729

Steiner, M., Philipps, S. P., Hermle, M., Bett, A. W., Dimroth, F., Jan. 2011. Validated front contact grid simulation for GaAs solar cells under concentrated sunlight. Progress in Photovoltaics: Research and Applications 19 (1), $73-83$.

URL http://onlinelibrary.wiley.com/doi/10.1002/pip.989/abstract

Sun, X., Raguse, J., Garris, R., Deline, C., Silverman, T., Alam, M. A., 2015. A physics-based compact model for CIGS and CdTe solar cells: from voltagedependent carrier collection to light-enhanced reverse breakdown. In: Photovoltaic Specialist Conference (PVSC), 2015 IEEE 42nd. IEEE, pp. 1-6.

URL http : / / ieeexplore . ieee .org / xpls / abs_all . jsp? arnumber $=$ 7355778

Szaniawski, P., Lindahl, J., Trndahl, T., Zimmermann, U., Edoff, M., May 2013. Light-enhanced reverse breakdown in $\mathrm{Cu}(\mathrm{In}, \mathrm{Ga}) \mathrm{Se} 2$ solar cells. Thin Solid Films 535, 326-330.

URL http : / / www . sciencedirect . com / science / article / pii / S0040609012011364

Sze, S. M., Ng, K. K., 2006. Physics of semiconductor devices. John wiley \& sons.

URL https : / / books . google $\cdot$ com/books?hl=en\&lr=\&id=o4unkmHBHb8C\& $\circ i=f$ nd \& $p g=P R 7 \& d q=$ Sze \& ot s $=w F w f 9 H A e 6 U \&$ sig $=$ AwPMdOGCde1zvyj $\mathrm{X} 1 \mathrm{jABj} 1 \mathrm{gitU}$

Vasko, A. C., Vijh, A., Karpov, V. G., Oct. 2014. Hot spots spontaneously emerging in thin film photovoltaics. Solar Energy 108, 264-273.

URL http : / / www . sciencedirect . com / science / article / pii / S0038092X14003570 
Westin, P.-O., Zimmermann, U., Stolt, L., Edoff, M., 2009. Reverse bias damage in CIGS modules. In: 24th European Photovoltaic Solar Energy Conference, 21-25 September 2009, Hamburg, Germany. WIP-Renewable Energies, pp. $2967-2970$.

URL http://www . diva-portal .org/smash/record.jsf ?pid=diva2: 249065 


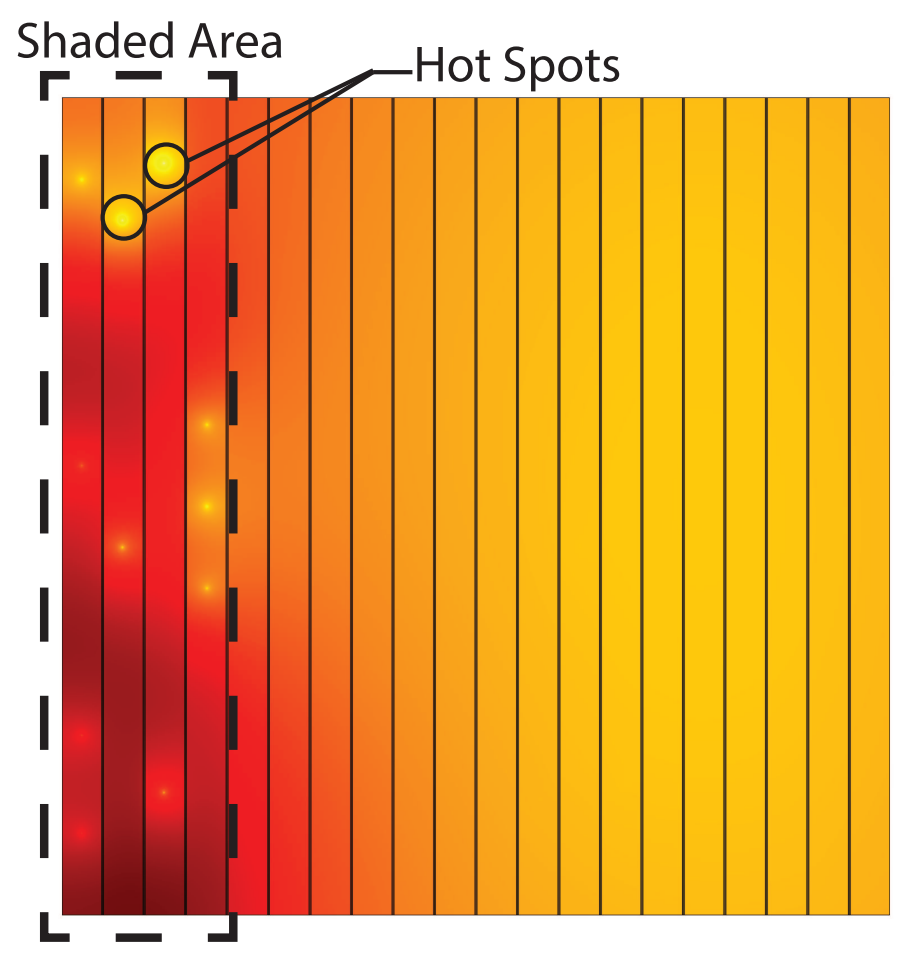

Monolithic Module Temperature

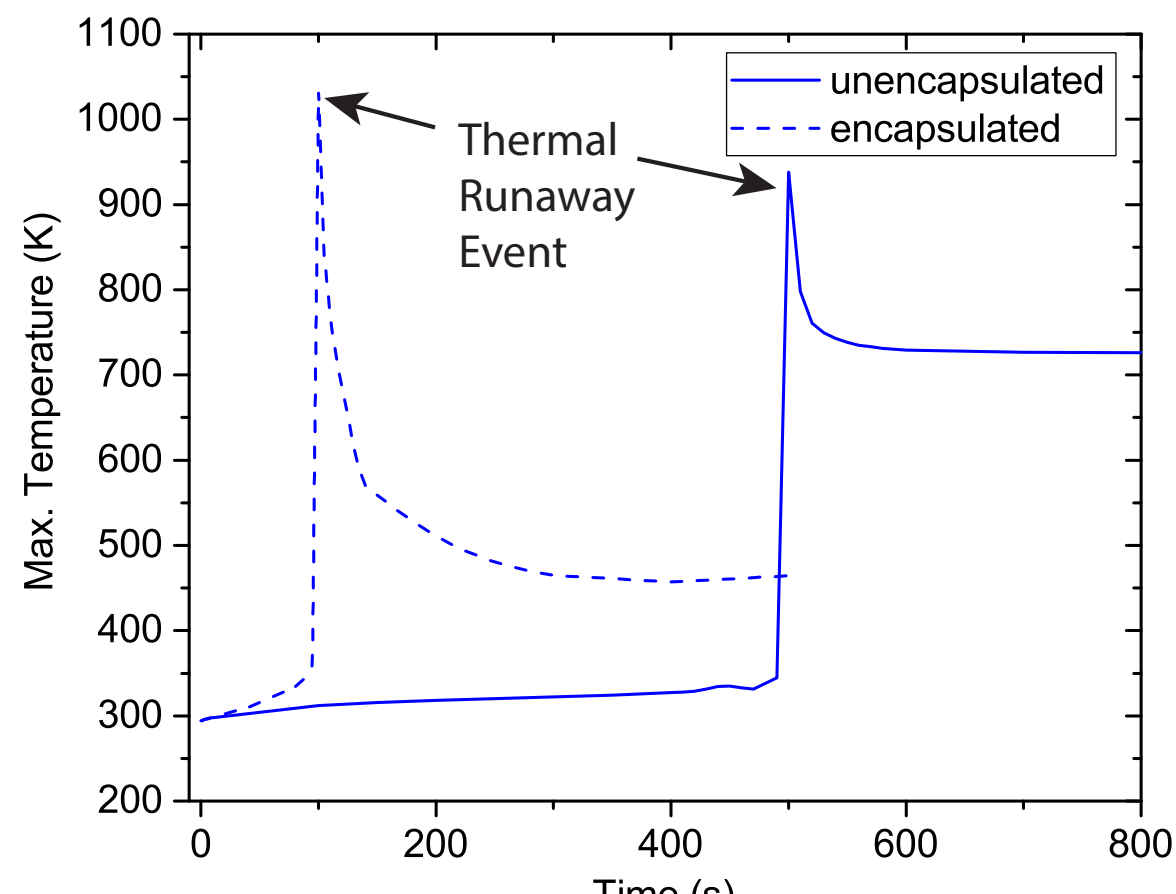

\section{Time (s)}

